\title{
High-resolution micro-computed tomography analyses of the abnormal trabecular bone structures in klotho gene mutant mice
}

\author{
T Yamashita ${ }^{1,3}$, Y-i Nabeshima ${ }^{2,3}$ and M Noda $^{1}$ \\ ${ }^{1}$ Department of Molecular Pharmacology, Medical Research Institute, Tokyo Medical and Dental University, Tokyo, Japan \\ ${ }^{2}$ Department of Pathology and Tumor Biology, Graduate School of Medicine, Kyoto University, Kyoto, Japan \\ ${ }^{3}$ Core Research for Evolutional Science and Technology, JST, Saitama, Japan \\ (Requests for offprints should be addressed to M Noda, 3-10, Kanda-Surugadai 2-Chome, Chiyoda-ku, Tokyo, 101-0062, Japan; \\ E-mail: noda.mph@mri.tmd.ac.jp)
}

\begin{abstract}
Inactivation mutation of the recently discovered klotho gene in mice causes a syndrome resembling aging. Manifestations include short life span, atherosclerosis, gonadal atropy, skin atropy, emphysema, ataxia and ectopic calcification. These mice also exhibit abnormally high bone density in the epiphyses of their tibiae based on X-ray and histological analyses. However, microstructures of the trabecular bones in arbitrary twodimensional planes or three-dimensional regions are difficult to analyze by these techniques. Therefore, we applied high resolution micro-computed tomography $(\mu \mathrm{CT})$ to characterize the micro-structural abnormality in the trabecular bone in long bones as well as in vertebrae of four- to six-week-old klotho mutant mice. Twodimensional $\mu \mathrm{CT}$ analyses in the mid-sagittal plane as well as three-dimensional $\mu \mathrm{CT}$ analyses indicated that the trabecular bone volume fraction measured in the proximal metaphyses of the tibiae was increased more than twofold
\end{abstract}

in klotho mutant mice compared with the wild-type mice. Similarly, the trabecular bone area fraction in the midsagittal plane of the lumbar vertebral bodies was also increased by about $80 \%$ at the proximal and distal ends. No significant difference was observed with regard to the cortical thickness in the mid-shaft of femora between klotho mutant and wild-type mice. Three-dimensional $\mu \mathrm{CT}$ analyses also indicated that the trabecular number and thickness of the proximal metaphyses of the tibiae were increased by about $80 \%$ and $300 \%$ respectively in the klotho mutant mice, while trabecular separation was $60 \%$ less in klotho mutant mice compared with the wild-type mice. These quantitative $\mu \mathrm{CT}$ analyses indicate that the inactivation of klotho gene expression results in an increase in three-dimensional bone volume fraction, number and thickness of the trabecular bones in these mice.

Journal of Endocrinology (2000) 164, 239-245

\section{Introduction}

Using insertional mutagenesis, the klotho gene was identified by Kuro-o et al. (1997) to be the gene whose inactivation causes a syndrome resembling aging. The Klotho protein predicted from its cDNA sequence is a membrane-bound enzyme (Kuro-o et al. 1997). One of the isoforms of the Klotho protein lacks its transmembrane domain, suggesting that there could be a soluble form (Matsumura et al. 1998, Shiraki-Iida et al. 1998). The klotho gene is abundantly expressed in kidney and slightly expressed in brain. In addition, very faint expression of the klotho gene is detectable by reverse transcription-PCR in tissues such as ovary, testis, skeletal muscle and pancreas but not in bone (Kuro-o et al. 1997).

One of the striking features of the klotho mutant mice is the elongation of trabecular bone in the histology of the metaphyseal regions of tibiae compared with those of the wild-type mice (Yamashita et al. 1998, Yamashita et al.
2000). However, it is difficult to obtain information on the three-dimensional or tomographic micro-structures by conventional X-ray examination and histological studies. As the precise function of Klotho protein is not known, it is important to characterize, in detail, the phenotypes of the mutant mice, including that in bone. Therefore, we applied micro-computed tomography $(\mu \mathrm{CT})$ to examine this abnormality in three-dimensional and tomographic micro-structures of the bones of the klotho mutant mice.

\section{Materials and Methods}

\section{Animals}

The klotho mutant mouse line was generated as described previously (Kuro-o et al. 1997). Genotypes were determined by Southern blot analyses using probes to detect the inserted plasmid located adjacent to the klotho gene locus. For experiments, four- to six-week-old mice were used. 
Five klotho mutant mice (average ages and sizes (length from the snout to the caudal end of the pelvis): $5 \cdot 8$ weeks and $67 \cdot 2 \mathrm{~mm}$ respectively) and five wild-type mice (average ages and sizes: $5 \cdot 2$ weeks and $70 \cdot 2 \mathrm{~mm}$ respectively) were used. All experiments were carried out according to the guidelines for animal welfare.

\section{Micro-CT analyses}

Bones of either klotho mutant or wild-type littermate mice were used for $\mu \mathrm{CT}$ analysis. Lumbar vertebrae, femora and tibiae were fixed in either $70 \%$ ethanol or $4 \%$ paraformaldehyde prior to the analyses. Two-dimensional analysis was conducted using $\mu \mathrm{CT}$ apparatus 'Musashi' (Nittetsu-ELEX, Tokyo, Japan). This apparatus consists of an X-ray tube operated at $40 \mathrm{kV}$ with a minimum focus of $6 \times 8$ microns and an X-ray detector linked to a high vision camera with $1024 \times 1024$ elements. For quantification, Luzex-F image analyzing system was used (Nireco, Tokyo, Japan). The procedure for obtaining a binary image is based on the auto setting functions provided in the image analyzer system of Nireco (using the pixel size of the non-bone area vs that of the bone area to obtain the threshold for the binary image) or the one provided in Scanco $\mu$ CT20 (the threshold value to obtain the binary image is given by the manufacturer, based on their calibration of the apparatus using mouse bone samples). To obtain two-dimensional (2D) $\mu \mathrm{CT}$ pictures the slices were set in the mid-sagittal plane. The slice thickness was 11 microns.

In setting the planes of section, any deviation of the sagittal section planes from the central axis of the bones, in other words, a shift of the section planes closer to the inner cortical wall of the bone marrow, would result in apparently higher values of bone area, since the density of trabeculae is greater nearer the cortex of bones than in the central area of the bone marrow. To avoid such an artifact, the planes of sections were set precisely in the central axis of the bones by monitoring the setting planes via X-ray scout view pictures.

For quantification, tomographic pictures of the midsagittal planes of the vertebral bodies and proximal tibiae were obtained. The trabecular bone area was measured in the tissue area $(0.7 \mathrm{~mm} \times 2.1 \mathrm{~mm})$ in the metaphyses of the proximal regions of tibiae adjacent to the growth plate. For vertebral bodies, the area of trabecular bones in the tissue area $(0.7 \mathrm{~mm} \times 1.4 \mathrm{~mm})$ of the region adjacent to the growth plate of the sixth lumber vertebrae was measured. The concentric artifact lines outside the bones in the pictures were excluded from the quantification (see Figs 1B,C, 2B,C). The concentric lines inside the area of bones were partially included into the quantification as noise; however, the levels of this background noise were less than the signals of most bones and were similar among all the bones measured in our experiments, and, therefore, did not affect the comparison between the klotho mutant mice bones and the wild-type mice bones. For the analyses of femoral head and neck regions, $2 \mathrm{D}-\mu \mathrm{CT}$ pictures of the frontal section planes including the longitudinal axis of the neck were taken, and cancellous bone area in the head was measured.

Three-dimensional (3D) $\mu \mathrm{CT}$ analysis of the epiphyseal regions of tibiae was conducted using a desk-top 3D- $\mu \mathrm{CT}$ analysis system ( $\mu$ CT20, Scanco Medical, Zurich, Swiss) (Ruegsegger et al. 1996). The spatial resolution is approximately $28 \mu \mathrm{m}$. Software based on an IPL package (Muller et al. 1994) equipped in the Scanco apparatus was used for the analysis of the parameters including trabecular bone volume, trabecular bone surface, trabecular numbers and trabecular separation. For $3 \mathrm{D}-\mu \mathrm{CT}$ analysis, measurement was conducted in a globular region with a diameter of $0.54 \mathrm{~mm}$ in the metaphysis adjacent to $(0.36 \mathrm{~mm}$ away from) the growth plate. The procedure for obtaining a binary image is based on the auto setting functions equipped in the image analyzer system of Nireco (using the pixel size of the non-bone area vs that of the bone area to obtain the threshold for the binary image) or the one equipped in Scanco $\mu \mathrm{CT} 20$ (the threshold value to obtain the binary image is given by the manufacturer, based on their calibration of the apparatus using bone samples).

Thresholds for the measurements were set at 275 (for Scanco system) and 140 (for Luzex-F) for 3D- and 2D-analyses respectively.

\section{Statistical analysis}

All parameters in the results were expressed as means \pm S.E.M. Differences between the mean values were evaluated by Mann-Whitney's U-test and significance was accepted when the $P$ value was less than $0 \cdot 05$.

\section{Results}

Two-dimensional $\mu \mathrm{CT}$ was conducted using mid-sagittal tomographs of the vertebral bodies (sixth lumbar vertebrae). The slice plane was precisely adjusted by using monitoring X-ray pictures as indicated in Fig. 1A. The tomography indicated that the trabecular bone area at both ends of the vertebral bodies of klotho mutant mice (Fig. 1B) were greater than those in the wild-type animals (Fig. 1C). Quantification of the trabecular bone fraction bone area/ total area (BAr/TAr) in the lumbar vertebrae indicated an approximately $80 \%$ increase in klotho mutant mice compared with the wild-type mice $(64 \cdot 0 \pm 3 \cdot 4 \%$ vs $36.9 \pm 3.4 \%$ respectively, $P<0.01$, see Fig. 3$)$.

Similarly, $2 \mathrm{D}-\mu \mathrm{CT}$ analyses indicated that the trabecular bone area in the mid-sagittal planes (Fig. 2A) in the metaphyseal regions of the tibiae in klotho mutant mice (Fig. 2B) was also greater than that in the wild-type (Fig. 2C). Quantification of the digitized images indicated that the trabecular bone area fraction in the proximal tibial 

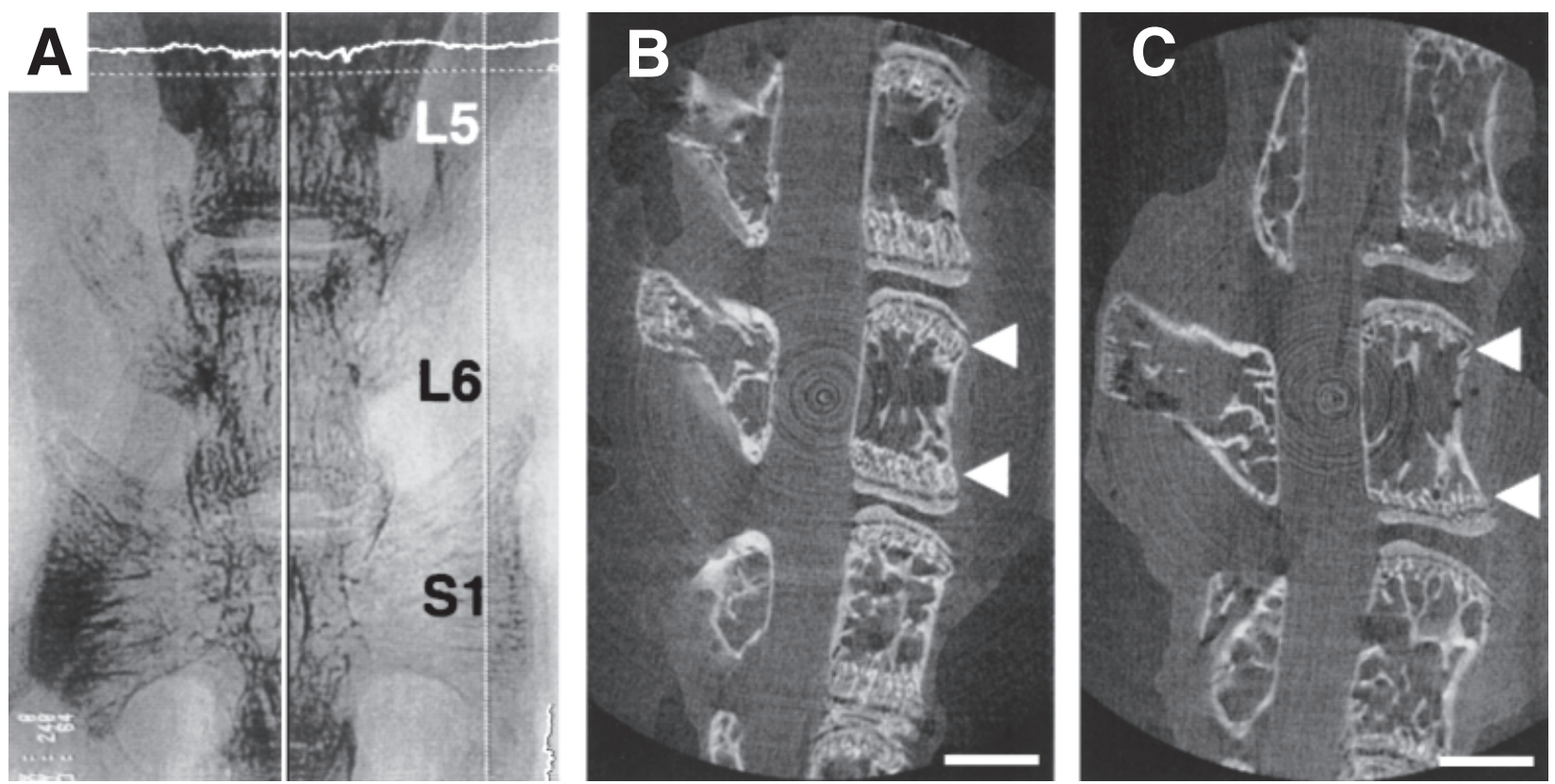

Figure 1 Two-dimensional tomographs of the vertebral bones of klotho mutant and wild-type mice. Trabecular bone area in the vertebral bones of klotho mutant (B) and wild-type mice (C) was measured within the mid-sagittal planes of the sixth vertebrae (L6) bodies (A). The line in (A) indicates the plane of the tomographic section. L5, L6 and S1 indicate the fifth and sixth lumbar and the first sacral vertebral bodies respectively. Five klotho mutant mice and five wild-type mice were used. Arrow heads: trabecular bone at the ends of vertebral body. Bar: $1.0 \mathrm{~mm}$.

metaphyses of the klotho mutant mice was over twofold greater than the corresponding regions of the tibiae of wild-type mice $(33 \cdot 2 \pm 5 \cdot 4 \%$ vs $12 \cdot 4 \pm 4 \cdot 7 \%$ respectively, $P<0 \cdot 01$, Fig. 3). On the other hand, cortical bone thickness in the mid-shaft of femora in both klotho mutant and wild-type mice was not significantly different (Fig. 4A and B).

Although the regions in the femoral neck of the mice are extremely small and it is hard to obtain histological sections in planes containing the central long axis of the anteverted neck, $\mu \mathrm{CT}$ enabled us to analyze easily the bone structures in this particular section plane (Fig. 5). As shown in Fig. 5A, the planes of tomographic slices were adjusted to parallel the long axis of the femoral neck and sections in the center of the femoral neck were obtained (Fig. 5B and C).

As shown in Fig. 6, 3D-reconstruction of the images of the proximal regions of the tibiae of klotho mutant mice
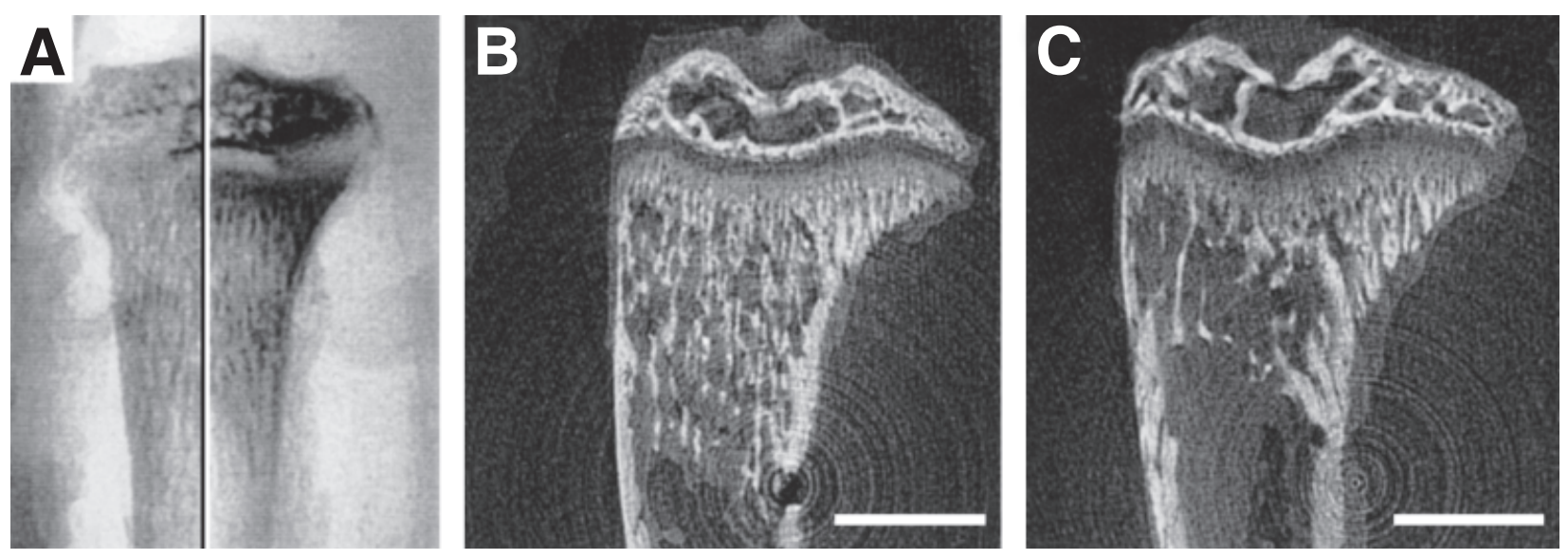

Figure 2 Two-dimensional tomographs of the proximal regions of tibiae: (B) klotho mutant mice; (C) wild-type mice. Trabecular bone area was measured within the sagittal plane of the tibia. The line in (A) indicates the plane of the tomographic section. Five klotho mutant mice and five wild-type mice were used. Bar: $1.0 \mathrm{~mm}$. 


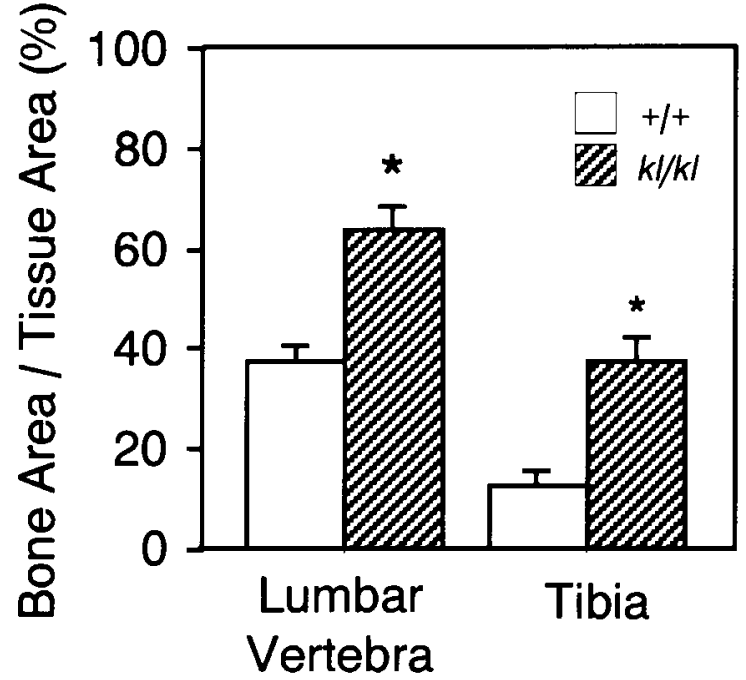

Figure 3 The trabecular bone area fraction, obtained by 2D-morphometry, of the sixth lumbar vertebrae and proximal tibiae in klotho mutant mice and in control mice. The trabecular bone area fraction in klotho mutant mice and the control mice was measured as described in Materials and Methods. The trabecular bone area fraction of both bones increased in klotho mutant mice. Five klotho mutant mice and five wild-type control mice were used. Open bars, control mice $(+/+)$; shaded bars, klotho mutant mice $(k l / k l)$. Results are means \pm S.E.M. ${ }^{*} P<0 \cdot 01$ compared with respective control.

indicated the high density of the trabecular bones in the metaphysis (Fig. 6A, closed arrow). 3D-quantification of the trabecular bone volume fraction, bone volume/total volume $(\mathrm{BV} / \mathrm{TV})$ in the tibial metaphyses of the klotho mutant mice indicated about a fivefold increase compared with the wild-type mice $(48 \cdot 3 \pm 1 \cdot 5 \%$ vs $8 \cdot 8 \pm 3 \cdot 2 \%$ respectively, $P<0 \cdot 01$, Fig. $7 \mathrm{~A}$ ).

We also found that the 3D-parameters were affected by the inactivation mutation in the klotho gene. Trabecular number (Tb.N) increased about twofold $(10 \cdot 6 \pm 0 \cdot 4$ vs $5.7 \pm 0.8$ per $\mathrm{mm}$ respectively, $P<0.01$, Fig. $7 \mathrm{~B}$, left). Moreover, trabecular thickness ( $\mathrm{Tb} . \mathrm{Th}$ ) in the tibiae of klotho mutant mice was three times more than that of wild-type $\quad(46 \cdot 0 \pm 1.3$ microns vs $13.2 \pm 3.7$ microns respectively, $P<0 \cdot 01$, Fig. $7 \mathrm{~B}$, center). Trabecular separation (Tb.Sp) was reduced by about $60 \%$ in klotho mutant mice compared with the wild-type mice $(49 \cdot 5 \pm 3 \cdot 0$ microns vs $182 \cdot 2 \pm 31 \cdot 1$ microns respectively, $P<0 \cdot 01$, Fig. $7 \mathrm{~B}$, right) in the proximal metaphyseal regions of the tibiae.

\section{Discussion}

Micro-CT based quantitative evaluation of the abnormal morphology of the bones in klotho mutant mice revealed the following characteristics. First, three-dimensional
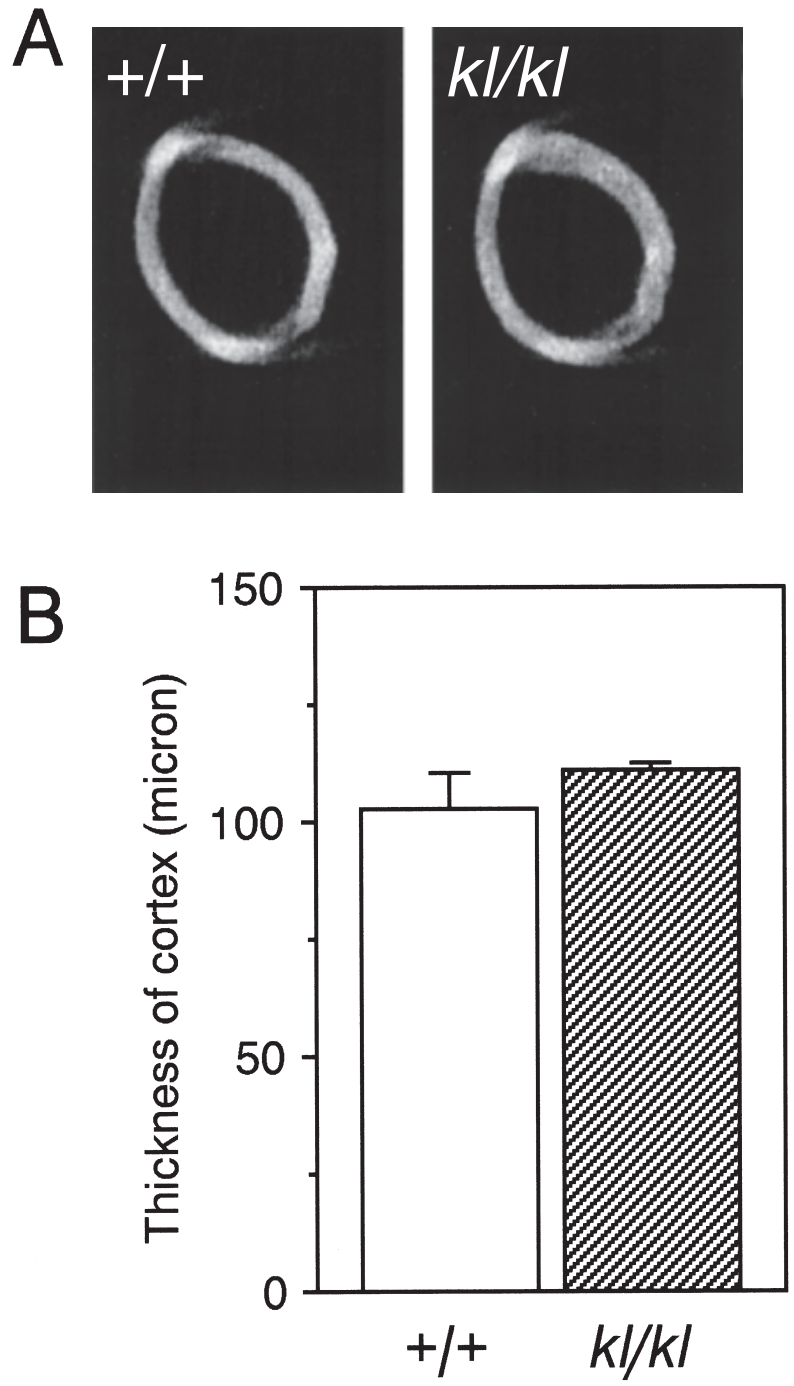

Figure 4 Cortical thickness at the mid-shaft of femora in klotho mutant and wild-type mice. (A) Two-dimensional images of the plane perpendicular to the long axis at the mid-shaft of femora of klotho mutant mice $(k l / k l)$ and wild-type mice $(+/+)$. (B) Cortical thickness was measured as described in Materials and Methods. Cortical thickness of femora in the klotho mutant and wild-type mice was not significantly different. Five klotho mutant mice and five wild-type mice were used. Open bars, control mice; shaded bars, klotho mutant mice. Results are means \pm S.E.M.

trabecular bone volume in tibiae was increased about fivefold. Secondly, trabecular number and trabecular thickness were increased in the metaphyses of tibiae of the klotho mutant mice (Fig. 7). The bone area in the vertebral bodies of klotho mutant mice analysed based on $2 \mathrm{D}-\mu \mathrm{CT}$ data was also $80 \%$ more than in the control wild-type mice (Figs 1 and 3). Overall, these three- and two-dimensional analyses indicate that the volume of the trabeculae in the metaphyseal regions of the bones 

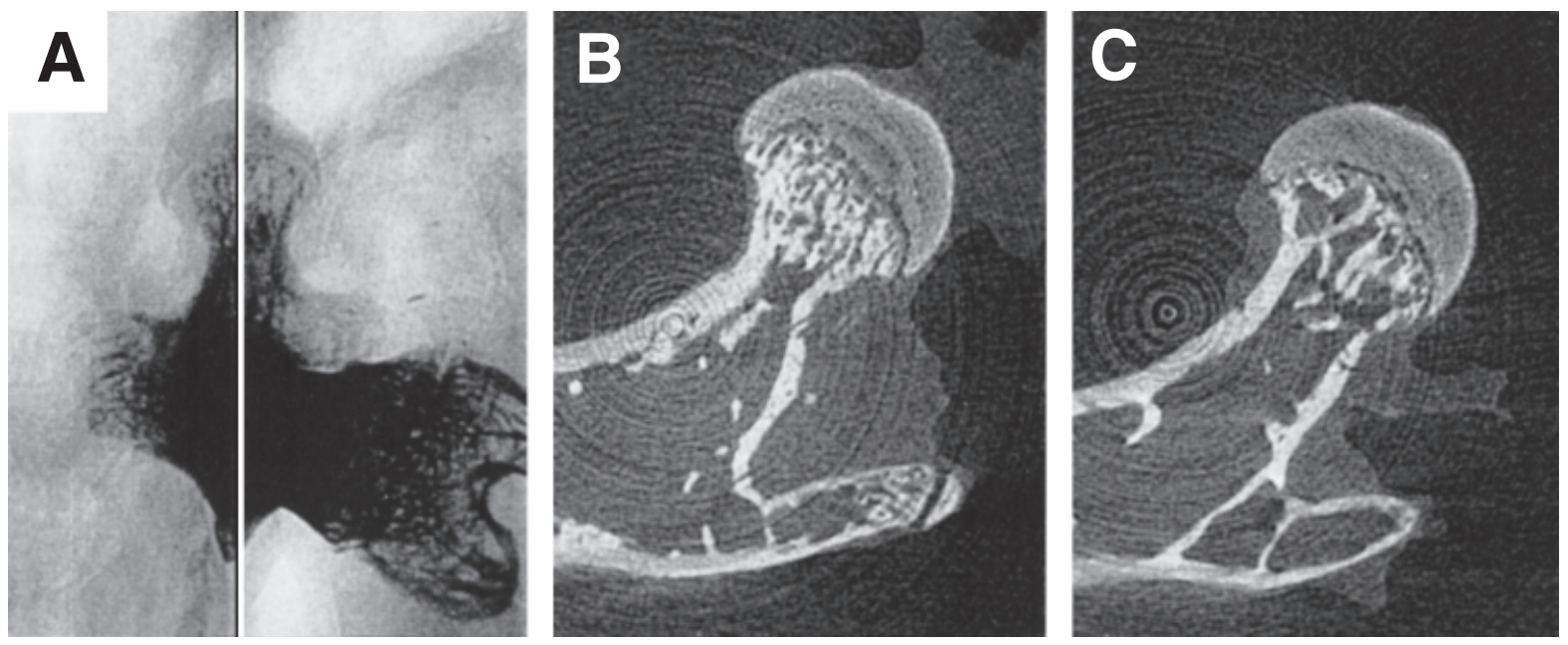

Figure 5 Two-dimensional tomographs of the femoral neck. The trabecular bone area in the femoral head of klotho mutant (B) or wild-type (C) mice was measured in the plane containing the central axis of the neck. The plane of section is indicated by the line in $(A)$.

of klotho mutant mice is more than that found in the wild-type mice.

We previously examined the bones of klotho gene mutant mice histologically. Although these previous

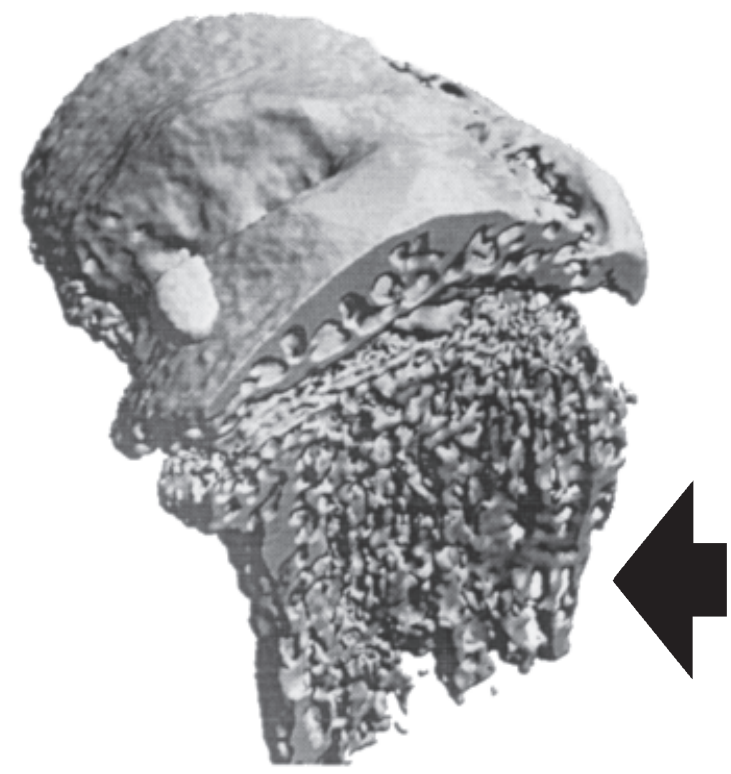

\section{(A) klotho mutant}

histological examinations revealed elongation of the trabecular bones (Yamashita et al. 1998, Yamashita et al. 2000), it was difficult to quantitate and to compare the amounts of trabecular bones in klotho mutant and

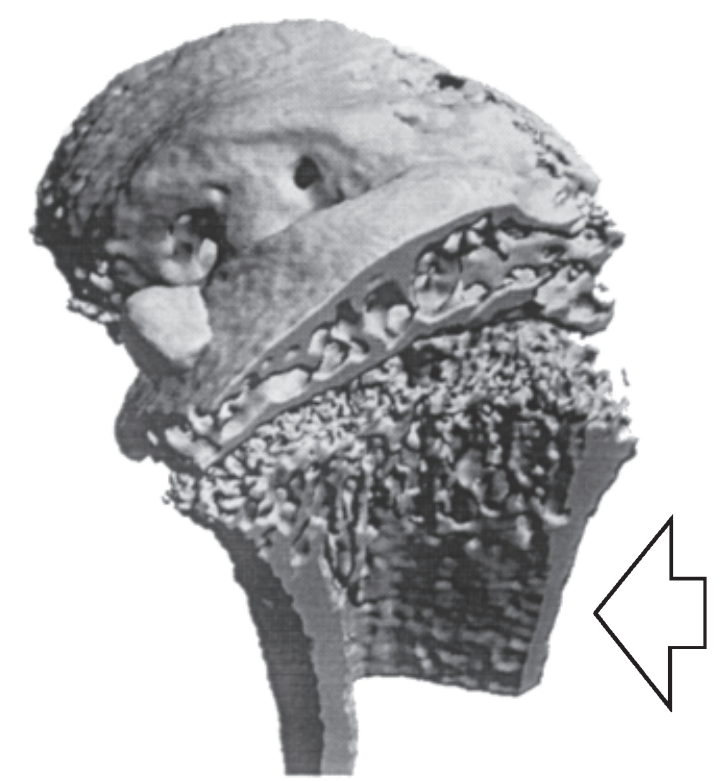

(B) Wild-type

Figure 6 Three-dimensional structures of the metaphyseal cancellous bones of the tibiae. Three-dimensional micro-computed tomographs were obtained by scanning the proximal regions of the tibiae of klotho mutant $(\mathrm{A})$ and wild-type (B) mice. The two-dimensional data were first obtained as a function of the distance from the epiphyses of the tibia and were reconstituted to construct 3D-structures. Closed arrow indicates the elongation of trabecular bones in the proximal region of tibia in the klotho mutant mouse. Open arrow indicates the corresponding region in the wild-type mouse tibia. 
A

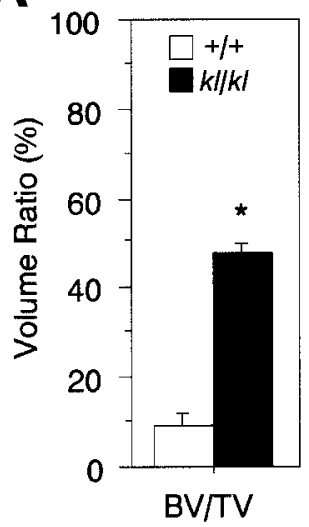

B

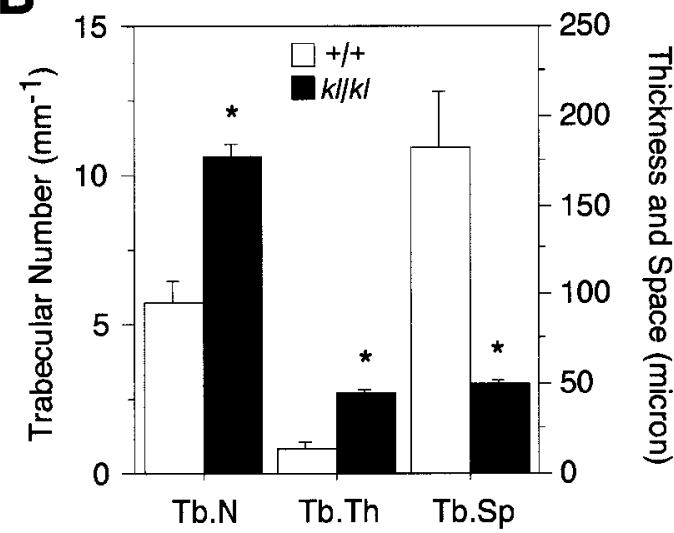

Figure 7 Three-dimensional morphometry of the trabecular bones in the tibia of klotho mutant (solid bars, $k l / k l$ ) and wild-type (open bars, $+/+$ ) mice. (A) Trabecular bone volume fractions (BV/TV) in the proximal regions of the tibiae of klotho mutant mice and that of wild-type mice were measured as described in Materials and Methods. (B) The number and thickness of trabecular bones and the space between trabecular bones were analyzed as described in Materials and Methods. Five klotho mutant mice and five wild-type mice were used. BV, trabecular bone volume; Tb.N, number of trabecular bones per unit length; Tb.Th, thickness of trabecular bones (microns); Tb.Sp, space between trabecular bones (microns). Results are means \pm S.E.M. ${ }^{*} P<0 \cdot 01$ compared with respective wild-type mice.

wild-type mice since the planes of the histological sections could not be precisely adjusted to the sharply central sagittal planes of the long bones. Although setting of the section plane is difficult, if not impossible, in histological examination, these problems in quantification were solved by the employment of high-resolution microcomputed tomography. Using this method, one can measure the bone volume quantitatively either twodimensionally or three-dimensionally without cutting or breaking the bones. More importantly, one can align the sagittal section planes for measurement precisely along the central axes of the vertebral bodies, the long bones such as the tibiae, and of very small regions such as the femoral neck of mice (Figs 1A, 2A and 5A). The digitized data of the images of the bones were quantified using an image analyzer.

Since the growth of klotho mutant mice is retarded after weaning, it is important to know whether our observations described in this paper on the klotho phenotype in the trabecular bone are due simply to the retardation of growth of these mice or are still observed regardless of the size of the animals. At the ages used in our experiments (average ages for wild-type and klotho mutant mice were 5.2 and 5.8 weeks respectively), the sizes of the klotho mutant mice were about 5\% less than those of wild-type mice, and the body weights of the klotho mutant mice were about $20 \%$ less than those of the wild-type. To address this point, we compared the morphology of trabecular bones in vertebrae and tibiae using klotho mutant mice and wild-type animals with similar body weights. These analyses revealed that morphological changes were still observed, similar to the data shown in Figs 1 and 2, even when the body weights of the animals were adjusted (data not shown).

Additionally, we examined the 2D $\mu \mathrm{CT}$-based bone area in a separate set of 10 wild-type mice and 7 klotho mutant mice. These 17 mice were totally different from the 10 mice described in this paper. Quantitation of the trabecular bone area (BAr/TAr) in the mid-sagittal plane of the femora (wild type, $n=10,5 \cdot 4 \pm 0 \cdot 6 \%$ vs klotho mutant mice, $n=7,10 \cdot 5 \pm 1 \cdot 1 \%, P<0 \cdot 05)$ indicated that the results are similar to those presented in this paper.

It is interesting to note that the thickness and diameter of the cortical bones are similar in klotho mutant mice and in wild-type mice even though the body sizes and body weights differ by about 5 and 20\% respectively. Although we do not have any explanation for this phenomenon, it could reflect relatively low levels of cortical bone turnover compared with that of trabecular bone and/or it could be influenced by the similarity in the growth of klotho mutant and wild-type mice up to the time of weaning, by which time the sizes of most of the bones are similar.

In our previous report, we found that the number of tartrate-resistant acid phosphatase (TRAP)-positive cells per area of the histological sections in the trabecular bone area was less than that of normal mice, suggesting a reduction in bone resorption activity in vivo (Yamashita et al. 1998). Whether our observations presented in this paper also suggest impairment in bone resorption in klotho mutant mice in vivo will have to be elucidated in the future. 
The increase in the trabecular bone volume in the klotho mutant mice was first reported in previous papers by Kuro-o et al. (1997), Yamashita et al. (1998) and Yamashita et al. (2000). Our present data further characterize the structural features of the trabecular bone of klotho mutant mice. Although klotho mutant mice reveal numerous aging-related features in various tissues including aorta, brain, skin, gonad, lung and bone, it is not known how the Klotho protein is functioning in these tissues. Therefore, it is important to identify how the Klotho protein acts in these affected organs, including bone. However, at this time it is difficult to obtain Klotho protein product and no powerful in vitro assay systems are available to examine the direct actions of klotho gene product in the cells of any of the above mentioned tissues. Therefore, detailed characterization of any of the phenotypes in klotho mutant mice should be pursued to find a clue to establish a direct and sensitive assay for Klotho protein function. Accumulation of trabecular bone in klotho mutant mice, as characterized by our $\mu \mathrm{CT}$ examination, seems to be in apparent contrast to the osteopenia in the diaphyses described in these mice. Although no clear explanation is yet available, this could be due to the difference in bone metabolism between human and rodents, as human growth plates are closed and bones stop growing after adolescence, while in rodents the growth plates do not close and continue to exist during the entire life of the animal, even after sexual maturation. Nonetheless, the identification of the mechanism(s) which gives rise to the interesting and clearly existing abnormality in the bones of klotho mutant mice offers the possibility of helping us to understand the function of the klotho gene product and, hopefully, the mechanisms of age-related bone loss in humans.

The morphological parameters examined in this study reflect more the quantity rather than the quality of the bones. To evaluate the detailed structural aspect of the trabecular bone, parameters which are more related to the structure of the bone, such as numbers of node and strut termini, trabecular pattern factors, marrow space star volume, trabecular star volume, mean intercept length or structural model index, have been measured (Hildebrand et al. 1999). It is intriguing and important to know how these parameters would detect the abnormality of the bones in klotho mutant mice.

The major advantage of the $3 \mathrm{D}-\mu \mathrm{CT}$ analysis is to obtain values that are the accumulated data of the planes analyzed by $2 \mathrm{D}-\mu \mathrm{CT}$. The merit of $2 \mathrm{D}-\mu \mathrm{CT}$, on the other hand, is to offer information on the bones in any plane of section that investigators would like to evaluate, while its disadvantage is apparently that it is not able to give information on the accumulated data of all the slices. Therefore, both $3 \mathrm{D}-\mu \mathrm{CT}$ and $2 \mathrm{D}-\mu \mathrm{CT}$ have their merits and it must be determined in the future which technique is the best to evaluate particular features of the bones.

Overall, klotho mutant mice demonstrate osteopetrotic phenotypes in their epiphyseal region after weaning.
Further molecular and cellular analyses on the role of Klotho protein in bone would shed light on new aspects of regulation of the maintenance of bone metabolism. For these molecular analyses to be possible, the high resolution $\mu \mathrm{CT}$ examination in klotho mutant mice, as presented in this report, provides an important base of morphological and structural information which would not have been available by conventional histological analysis alone.

\section{Acknowledgements}

We thank Dr M Kuro-o for providing us with klotho transgenic mice. This research was supported by grant-inaid received from the Ministry of Education (11152209, 11877357, 10044246, 0930734) and grants from CREST (Core Research for Evolutional Science and Technology) of Japan Science and Technology Corporation (JST), a grant from the 'Research for the Future' Program of the Japan Society for the Promotion of Science (JSPS) (96I00205), grants from Traffic Medicine Foundation, Interdisciplinary Cancer Research Foundation and Inamori Foundation, Inamori Foundation, Multidisciplinary Cancer Research Foundation, NASDA and Marine and Fire Insurance Foundation.

\section{References}

Hildebrand T, Laib A, Muller R, Dequeker J \& Ruegsegger P 1999 Direct three-dimensional morphometric analysis of human cancellous bone: microstructural data from spine, femur, iliac crest, and calcaneus. Journal of Bone and Mineral Research 14 1167-1174.

Kuro-o M, Matsumura Y, Aizawa H, Kawaguchi H, Suga T, Utsugi T, Ohyama Y, Kurabayashi M, Kaname T, Kume E, Iwasaki H, Iida A, Shirakiiida T, Nishikawa S, Nagai R \& Nabeshima Y 1997 Mutation of the mouse klotho gene leads to a syndrome resembling ageing. Nature $39045-51$.

Matsumura Y, Aizawa H, Shirakiiida T, Nagai R, Kuroo M \& Nabeshima Y 1998 Identification of the human klotho gene and its two transcripts encoding membrane and secreted klotho protein. Biochemical and Biophysical Research Communications 242 626-630.

Muller R, Hildebrand T \& Ruegsegger P 1994 A new method to analyse and display the three-dimensional structure of trabecular bone. Physical Medical Biology 39 145-164.

Ruegsegger P, Koller B \& Muller R 1996 A microtomographic system for the nondestructive evaluation of bone architecture. Calcified Tissue International 58 24-29.

Shiraki-Iida T, Aizawa H, Matsumura Y, Sekine S, Iida A, Anazawa H, Nagai R, Kuroo M \& Nabeshima Y 1998 Structure of the mouse klotho gene and its two transcripts encoding membrane and secreted protein. FEBS Letters 424 6-10.

Yamashita T, Nifuji A, Furuya K, Nabeshima Y \& Noda M 1998 Elongation of the epiphyseal trabecular bone in transgenic mice carrying klotho gene locus mutation which leads to a syndrome resembling aging. Journal of Endocrinology 159 1-8.

Yamashita T, Yoshitake H, Tsuji K, Kawaguchi N, Nabeshima Y \& Noda M 2000 Retardation in bone resorption after bone marrow ablation in Klotho mutant mice. Endocrinology 141 (In Press).

Received 11 June 1999

Revised manuscript received 12 August 1999 Accepted 31 August 1999 